\title{
CONHECIMENTO DOS ACADÊMICOS DE ENFERMAGEM SOBRE A TÉCNICA EM CATETERISMO VESICAL
}

\author{
Simone Shirasaki Orosco, Crisitiane Gracielle da Silva, Thatiana Killian de Almeida \\ Universidade do Oeste Paulista - UNOESTE, Curso de Enfermagem, Presidente Prudente, SP. e-mail: \\ simone.orosco@hotmail.com
}

\section{RESUMO}

O objetivo deste estudo foi identificar o conhecimento dos acadêmicos de enfermagem acerca do cateterismo vesical de longa permanência. Trata-se de uma pesquisa descritiva, transversal, com abordagem quantitativa. Foi aplicado um instrumento com questões de múltipla escolha aos graduandos de uma universidade do interior paulista. A amostra foi composta por 81 acadêmicos do sétimo termo, sendo a maioria do sexo feminino, faixa etária de 20 a 30 anos, estudantes do período noturno, solteiros, renda familiar de 1 a 4 salários mínimos, não possuem formação e nem experiência na área de enfermagem. As questões que apresentaram maior porcentagem de acertos foram sobre os materiais necessários para o procedimento $(93,8 \%)$, etapa que não faz parte do fim da técnica $(87,7 \%)$ e parte do processo do cateterismo (76,5\%). Conclui-se que boa parte dos estudantes tem conhecimento acerca da técnica, pois na maioria das questões mais de $50 \%$ do grupo apontou a alternativa correta.

Palavras-chave: Conhecimento, ensino, cateteres de demora, educação em enfermagem, estudantes de enfermagem.

\section{KNOWLEDGE OF NURSING ACADEMICS ON THE TECHNIQUE OF BLADDER CATHETERIZATION}

\begin{abstract}
The objective of this study was to identify nursing students' knowledge about long-term bladder catheterization. This is a descriptive, cross-sectional research with a quantitative approach. An instrument with multiple choice questions was applied to graduates of a university in the interior of São Paulo. The sample consisted of 81 students from the seventh term, most of them female, 20 to 30 years old, night students, singles, family income of 1 to 4 minimum salaries, no training and no experience in the area of nursing. The questions that presented the highest percentage of correct answers were the materials needed for the procedure (93.8\%), a step that is not part of the end of the technique $(87.7 \%)$ and part of the catheterization process $(76.5 \%)$. It is concluded that most of the students have knowledge about the technique, since in most of the questions more than $50 \%$ of the group pointed out the correct alternative. Keywords: Knowledge, teaching, indwelling catheter, nursing education, nursing students.
\end{abstract}

\section{INTRODUÇÃO}

O cateterismo vesical é um procedimento invasivo que requer técnica asséptica, e que consiste na introdução de um cateter estéril por meio do meato uretral até a bexiga e é conectado a um coletor também estéril, visando o alivio urinário do paciente através da cateterização vesical de longa permanência ou de alivio imediato. Sua utilização pode ser diagnóstica ou terapêutica ${ }^{1}$.

Este procedimento é utilizado para obtenção de um fluxo continuo ou de alivio da diurese dos pacientes com incontinência ou obstrução vesical, mensuração do débito urinário em pacientes críticos com instabilidade 
hemodinâmica, coleta de urina não contaminada para cultura e em pacientes prostrados, irrigação vesical em pacientes pós-cirúrgicos de bexiga ou próstata. Além disso, tem indicações para auxiliar na cicatrização de feridas abertas sacrais ou perineais em pacientes incontinentes, facilitar a continência e manter a integridade da pele (em casos de insucesso com métodos conservadores), melhorar o conforto em cuidados paliativos no final da vida, se necessário e tratamento da incontinência intratável ${ }^{2,3}$.

É muito importante que os profissionais da saúde saibam que o paciente deve ser cateterizado somente se houver necessidade e será realizado pela equipe por meio de um Enfermeiro ou Médico. A responsabilidade de instalar o cateterismo vesical de longa permanência cabe a eles, porque tal procedimento é uma das portas que mais leva a infecção urinária no ambiente hospitalar ${ }^{4}$.

A resolução COFEN no 0450/2013 considera que a execução do procedimento de cateterismo vesical requer ações que possam garantir que somente o profissional enfermeiro treinado faça a inserção dos dispositivos urinários, selecionando o cateter de menor calibre possível para a drenagem adequada, a fim de minimizar ocorrências de trauma, bem como identifique e monitore os grupos de pacientes susceptíveis a Infecção do Trato Urinário (ITU) ${ }^{5}$.

Assim, as complicações pelo uso do cateter vesical de longa permanência podem ser classificadas em infecciosas e traumáticas. As lesões uretrais e vesicais são as mais comuns pelo uso do cateter, e as complicações infecciosas geralmente estão associadas à invasão por microrganismo através de lesões na mucosa e bexiga que podem ocasionar abcessos e consequentemente fístulas ${ }^{6}$.

Igawa et al. ${ }^{7}$ acrescentam como complicações a incompetência do esfíncter uretral, formação de cálculos na bexiga, câncer na bexiga e reações de hipersensibilidade.

A infecção do trato urinário corresponde a mais de $30 \%$ de todas as infecções relacionadas à assistência à saúde (IRAS), ficando em sua totalidade relacionada à instrumentação do trato urinário. A infecção pode surgir em 1 a $2 \%$ dos pacientes submetidos ao cateterismo urinário intermitente, e em 10 a $20 \%$ dos pacientes submetidos ao cateterismo de longa permanência ${ }^{8}$.

A maioria das infecções do trato urinário é causada por uma única espécie bacteriana, sendo a Escherichia coli responsável por pelo menos $80 \%$ dos casos de cistite e de pielonefrite. Observa-se uma variedade de microrganismos que podem causar infecções do trato urinário de origem hospitalar, incluindo os seguintes gêneros: Pseudomonas e Staphylococcus. Ainda, entre as infecções adquiridas no ambiente hospitalar, as sepses por Staphylococcus aureus são responsáveis por elevada morbidade e mortalidade ${ }^{3,9}$.

Nesse contexto, as Infecções do Trato Urinário em alguns hospitais são mais frequentes que a pneumonia nosocomial e a bacteremia associada ao dispositivo intravascular. Verifica-se que a presença do cateter vesical de longa permanência na uretra retira os mecanismos de defesa intrínsecos do paciente tais como a micção e o esvaziamento eficiente da bexiga. Ademais, na Infecção do Trato Urinário geralmente ocorre a formação de biofilme tanto na parte interna como externa do cateter, que protege os microrganismo da ação de antimicrobianos e da resposta imune do hospedeiro ${ }^{7,9}$.

Várias organizações, atualmente, têm proposto e pesquisado recomendações para o controle da Infecção do Trato Urinário, com especial foco de atenção no uso do cateter urinário. No entanto, os índices de Infecções do Trato Urinário associadas ao uso do cateter ainda são preocupantes, acrescentando à prática clínica do enfermeiro indagações relacionadas com a escolha, modo de realização da técnica de inserção e manutenção do cateter, orientação da equipe de enfermagem, entre outros ${ }^{10}$.

O enfermeiro como líder responsável pela equipe detém conhecimento para desempenhar no seu exercício profissional, as avaliações e as indicações do uso contínuo ou permanente do cateter, assim como identificar as complicações e promover técnicas e intervenções para minimizar as complicações ${ }^{1}$.

Dessa forma, o profissional enfermeiro precisa desenvolver atividades educativas e estar num processo de educação permanente, visando - fortalecimento do vínculo profissional/usuário/serviço de saúde, a prática de procedimentos dentro dos parâmetros da biossegurança e técnicas assépticas, resultando assim na redução das infecções do trato urinário relacionadas à utilização de cateter vesical ${ }^{9}$.

Mazzo et al. ${ }^{11}$ afirmam que o método e os materiais para realizar a técnica não podem seguir apenas a prática rotineira, 
comprometendo pacientes e profissionais. É preciso buscar a padronização do cuidado aplicado ao cateterismo vesical, assim como a elaboração de protocolos institucionais devem ser reforçadas para melhorar a qualidade e a segurança no procedimento.

O processo de ensino e aprendizagem, focado na assistência, depende do desempenho visto que no ensino prático, a aprendizagem ocorre por meio do treino diário das habilidades. Contudo, um estudo mostrou que muitos estudantes do último semestre do curso de graduação em Enfermagem tinham pouca habilidade na prática clínica ${ }^{12}$.

Observa-se que os alunos do curso de enfermagem tem o sentimento de insegurança, medo, por sua inabilidade, quando estão diante de realizar procedimento que exija conhecimento e técnica mesmo em situações de laboratório. 0 medo aumenta mais quando está à beira do leito para realizar o procedimento, deixando-o transpassar ao cliente. Essas situações ocorrem, pois os estudantes têm dificuldade em lembrar os passos do cateterismo vesical e executar perfeitamente o procedimento ${ }^{12}$.

Além disso, as pesquisas demonstram que é pequeno o número de artigos produzidos pelos profissionais na área do cateterismo urinário, sendo necessário incrementar no corpo de conhecimento da enfermagem a produção de conhecimentos sobre o assunto para subsidiar as discussões, orientações e treinamento da equipe, com a finalidade de propor diretrizes atualizadas, protocolos inovadores e materiais adequados que podem oferecer segurança ${ }^{10}$. Desse modo, o objetivo deste estudo foi identificar o conhecimento dos estudantes de enfermagem acerca da técnica do cateterismo vesical de longa permanência.

\section{METODOLOGIA}

Trata-se de uma pesquisa descritiva, transversal, com abordagem quantitativa.

Os participantes desta pesquisa foram 81 estudantes do sétimo termo do curso de graduação em enfermagem. Participaram de atividades teóricas e praticas nas disciplinas de Assistência de Enfermagem Clínica e Cirúrgica.

$O$ estudo foi realizado em uma universidade do interior paulista, no curso de graduação em enfermagem, com quatro anos de duração e carga horária mínima de 4000 h, sendo oferecido semestralmente nos períodos diurno e noturno. O curso oferece atividades praticas em unidades básicas de saúde/estratégias saúde da família, hospital e no laboratório de habilidades de simulação.

0 instrumento para avaliação do conhecimento acerca do cateterismo vesical de longa permanência entre os graduandos de enfermagem foi validado por Balduíno ${ }^{12}$ e adaptado pelos pesquisadores.

A coleta de dados constou das seguintes etapas: (i) os estudantes do termo citado acima foram abordados na sala de aula pelos pesquisadores, no início da aula após autorização prévia do professor, onde os mesmos explicaram o objetivo da pesquisa; (ii) os interessados em participar da pesquisa receberam um termo de Consentimento Livre e Esclarecido que foi assinado e devolvido; (iii) foi entregue o instrumento de coleta de dados, preenchido e colocado dentro de uma caixa sem identificação.

Os dados foram organizados e analisados por meio de planilhas do Microsoft Excel 2010. Além disso, foram elaboradas tabelas para apresentação de frequências absoluta e relativa.

Os aspectos éticos da pesquisa foram conduzidos de acordo com a Resolução 466/12 do Conselho Nacional da Saúde após aprovação do Comitê de Ética em Pesquisa (CEP) da universidade sob $o$ protocolo CAAE $n$. 61986816.7.0000.5515.

Os resultados serão apresentados à coordenação do curso e aos docentes para possível reorganização e melhoria do processo de ensino e aprendizagem sobre as técnicas de enfermagem.

\section{RESULTADOS}

O perfil sóciodemográfico dos acadêmicos de enfermagem do sétimo termo do curso de graduação de uma universidade do interior paulista está apresentado na Tabela 1.

A maioria dos acadêmicos era do sexo feminino $(n=59,72,8 \%)$ na faixa etária entre 20 e 30 anos $(n=61,75,3 \%)$, sendo $64,2 \%(n=52)$ do período noturno. A renda familiar predominante foi de um a quatro salários mínimos ( $n=39$, $48,1 \%$ ) e o estado civil foi predominantemente de solteiros ( $n=56,69,1 \%)$. Quanto à formação na área de enfermagem, $72,8 \%(n=59)$ não possuem nenhuma formação e $80,2 \%$ ( $n=65$ ) não possuem nenhuma experiência na área. 
Tabela 1. Características sociodemográficas de acadêmicos de enfermagem do sétimo termo do curso de Enfermagem ( $n=81)$.

\begin{tabular}{|c|c|c|c|}
\hline Características & & $f$ & $\%$ \\
\hline \multirow[t]{2}{*}{ Sexo } & Masculino & 59 & 72,8 \\
\hline & Feminino & 22 & 27,2 \\
\hline \multirow[t]{5}{*}{ Idade (anos) } & $20-30$ & 61 & 75,3 \\
\hline & $31-40$ & 16 & 19,8 \\
\hline & $41-50$ & 2 & 2,5 \\
\hline & $51-60$ & 0 & 0,0 \\
\hline & Não respondeu & 2 & 2,5 \\
\hline \multirow[t]{3}{*}{ Período de estudo } & Diurno & 26 & 32,1 \\
\hline & Noturno & 52 & 64,2 \\
\hline & Não respondeu & 3 & 3,7 \\
\hline Renda familiar & 1 a 4 & 39 & 48,1 \\
\hline \multirow[t]{3}{*}{ (salários mínimos) } & 5 a 8 & 12 & 14,8 \\
\hline & Acima 8 & 2 & 2,5 \\
\hline & Não respondeu & 28 & 34,6 \\
\hline \multirow[t]{6}{*}{ Estado civil } & Solteiro & 56 & 69,1 \\
\hline & União estável & 5 & 6,2 \\
\hline & Casado & 10 & 12,3 \\
\hline & Divorciado & 5 & 6,2 \\
\hline & Viúvo & 0 & 0,0 \\
\hline & Não respondeu & 5 & 6,2 \\
\hline Formação na área & Técnico & 11 & 13,6 \\
\hline \multirow[t]{2}{*}{ da Enfermagem } & Auxiliar & 11 & 13,6 \\
\hline & Não tem & 59 & 72,8 \\
\hline Tempo de experiência & $<1$ & 0 & 0,0 \\
\hline \multirow[t]{4}{*}{ na Enfermagem (anos) } & $1-5$ & 6 & 7,4 \\
\hline & $6-10$ & 4 & 4,9 \\
\hline & $11-15$ & 6 & 7,4 \\
\hline & Não tem & 65 & 80,2 \\
\hline
\end{tabular}

Do total de 12 questões sobre a técnica de cateterismo vesical de longa permanência masculino, a média de acertos dos acadêmicos de enfermagem no questionário foi de 6,52 $\pm 1,54$.

$\mathrm{Na}$ Tabela 2 estão apresentadas as variáveis de avaliação do conhecimento dos acadêmicos de enfermagem sobre a técnica de cateterismo vesical.
Em relação aos materiais hospitalares necessários para o procedimento de cateterismo vesical de longa permanência, 93,8\% $(n=76)$ deles demonstraram ter conhecimento.

Sobre algumas providências que devem ser tomadas para realizar o procedimento em pacientes conscientes, somente $30,9 \% \quad(n=25)$ apontaram a orientação ao paciente quanto ao procedimento, obtenção do consentimento e 
verificação do horário da ultima micção. E sobre a sequência correta da técnica de cateterismo vesical de longa permanência, 59,3\% $(n=48)$ demonstram saber corretamente a técnica.

Acerca do conhecimento em relação às precauções para se evitar uma Infecção do Trato Urinário, $71,6 \% \quad(n=58)$ têm conhecimento, ou seja, realizam a técnica asséptica de inserção do cateter.

Com relação ao passo que não faz parte do processo de cateterismo vesical a maioria ( $n=62,76,5 \%$ ) conseguiu identificar e em relação à rotina de troca do cateter vesical, apenas $8,6 \%$ $(n=7)$ demonstraram conhecimento.

A maioria $(n=50,61,7 \%)$ optou pela alternativa correta sobre $\mathrm{o}$ processo de cateterismo vesical de longa permanência e apenas $17,3 \% \quad(n=14)$ dos acadêmicos de enfermagem demonstram conhecimento na sequência correta da lavagem externa para iniciar o cateterismo vesical no sexo masculino.
Grande parte $(n=51,63 \%)$ dos acadêmicos de enfermagem identificou e assinalou a alternativa que aponta colocar campo fenestrado depois da assepsia como não fazendo parte dos passos para realização do cateterismo vesical de longa permanência.

Foram listadas algumas etapas do procedimento de cateterismo vesical, $54,3 \%$ $(n=44)$ identificaram a alternativa incorreta e $25,9 \%(n=21)$ dos acadêmicos de enfermagem selecionaram a etapa final do procedimento que foi corretamente explicada, ou seja, a fixação da sonda na região supra púbica sem tracioná-la. Dentre algumas etapas listadas em relação ao cateterismo vesical, $87,7 \%(n=71)$ reconheceram que calçar luvas de procedimento não faz parte do fim da técnica.

Tabela 2. Variáveis de avaliação do conhecimento de acadêmicos de acadêmicos de enfermagem do sétimo termo do curso de Enfermagem $(n=81)$.

\begin{tabular}{lcc}
\hline Variáveis de avaliação do conhecimento & $f$ & $\%$ \\
\hline 1. Materiais hospitalares necessários para o procedimento de SVD* & 76 & 93,8 \\
2. Providencias que devem ser tomadas para realização do procedimento em & & \\
pacientes conscientes & 25 & 30,9 \\
3. Sequencia correta da técnica de SVD* & 48 & 59,3 \\
4. Primeiro passo para se evitar ITU & 58 & 71,6 \\
5. Parte do processo de SVD*, exceto & 62 & 76,5 \\
6. Assinale a alternativa correta - rotina de troca de sonda & 7 & 8,6 \\
7. Assinale a correta - processo de sondagem vesical de demora & 50 & 61,7 \\
8. Sequência correta da lavagem externa para iniciar a SVD* no sexo masculino & 14 & 17,3 \\
9. Passos que fazem parte da SVD*, exceto & 51 & 63,0 \\
10. Etapa incorreta do procedimento de SVD* no sexo masculino & 44 & 54,3 \\
11. Etapa final da SVD* correta & 21 & 25,9 \\
12. Etapa que não faz parte do fim da SVD* & 71 & 87,7 \\
\hline
\end{tabular}

* Sondagem vesical de demora.

\section{DISCUSSÃO}

Este trabalho verificou o conhecimento dos acadêmicos de enfermagem sobre a técnica em cateterismo vesical, sendo a maioria do sexo feminino, na faixa etária de 20 a 30 anos, estudantes do período noturno e solteiros, não possuem formação e nem experiência na área de enfermagem.

Estes dados estão de acordo com demais estudos em que há predominância de mulheres na graduação de enfermagem. Porém, quanto à idade, dois estudos apontam que a maioria dos estudantes tem idades entre 17 e 23 anos $^{13,14}$. Em relação ao período, no estudo realizado em duas faculdades do Rio de Janeiro, a instituição pública ofertava o curso somente em horário integral, e na particular era parcial (manhã, tarde ou noite), possibilitando ao graduando estar inserido no mercado de trabalho, sendo que $61,3 \%$ deles eram profissionais da área de saúde de nível médio $^{14}$. 
Quanto ao estado civil há uma prevalência de solteiros, que refletem a realidade da população brasileira feminina que priorizam a formação profissional e a inserção no mercado de trabalho ${ }^{14}$.

O mesmo estudo apresenta como renda familiar dos graduandos superior a cinco salários mínimos ${ }^{14}$, enquanto este trabalho evidenciou que a renda familiar dos estudantes de enfermagem é de um a quatro salários mínimos.

Em relação à avaliação do conhecimento dos acadêmicos de enfermagem sobre a técnica de cateterismo vesical, a questão $1 \mathrm{faz}$ abordagem sobre os materiais hospitalares necessários para o procedimento do cateterismo vesical e a maioria dos estudantes elencou a alternativa correta que apresentava a seringa de $10 \mathrm{~mL}$, luva estéril, sonda Foley e ampola de água destilada.

Em um estudo que verificou como o procedimento de inserção do cateter urinário de demora tem se dado na prática clínica da enfermagem, os entrevistados citaram os mesmos materiais utilizados para 0 procedimento dentre outros como a bolsa coletora, solução de clorexidina, bolas de algodão, cuba rim, gaze e fita adesiva ${ }^{11}$.

Os cateteres comuns (plástico, látex, silicone, silicone hidrogel, polivinil-PVC) são os mais utilizados nas instituições e o Guideline for Prevention of Catheter-Associated Urinary Tract Infections 2009 descreve que o silicone pode ser preferível a outros materiais de cateter para reduzir o risco de incrustação em pacientes cateterizados em longo prazo que têm obstrução frequente ${ }^{15}$. Ainda, pesquisas apontam o uso de cateteres revestidos com algum tipo de solução antisséptica ou antimicrobiana para redução de infecção do trato urinário, porém não há evidências suficientes para determinar o melhor tipo de cateter urinário, com vistas à prevenção de infecções ${ }^{8}$.

0 Guideline e outras pesquisas ${ }^{16}$ demostram que o uso de luvas esterilizadas e a adoção de rigorosa técnica asséptica devem ser observados sempre na realização de um cateterismo vesical no ambiente hospitalar para prevenção da infecção.

Já a seringa e água destilada são necessárias para insuflar o balonete da sonda, sendo para drenagem padrão em cateteres adultos o volume de $10 \mathrm{~mL}$ suficiente e $30 \mathrm{~mL}$ somente para uso pós-operatório².
As questões 2 e 5 trazem algumas providências que devem ser tomadas antes do procedimento em pacientes conscientes como verificar o horário da última micção do paciente, orientar o paciente quanto ao procedimento, obter consentimento do paciente consciente, orientado para iniciar a sondagem e como alternativa incorreta verificar horário da última evacuação.

O cateterismo é um procedimento invasivo que pode causar constrangimento, desconforto psicológico e impacto na autoimagem do paciente. Para garantir que o paciente esteja totalmente preparado para o procedimento, é preciso que o profissional de saúde informe o paciente sobre as razões e necessidade do mesmo e obter a permissão. Em muitas áreas da medicina, os pacientes são obrigados a assinar um formulário de consentimento que indica um entendimento sobre o evento $\mathrm{e}$ as possíveis complicações/problemas associados. Na Europa não é uma prática comum os pacientes fornecerem consentimento por escrito para o cateterismo. No entanto, é necessário que o consentimento verbal e o acordo sejam alcançados, além das informações relevantes registradas nas anotações médicas e de enfermagem no prontuário do paciente ${ }^{2,11,17}$.

Em um estudo que verificou a existência de fatores relacionados à padronização do cateterismo urinário, em uma cidade do interior do estado de São Paulo, mais da metade dos profissionais entrevistados não mencionou cuidados quanto à orientação, privacidade ou formas de humanização, demonstrando um perfil profissional de enfermagem apenas técnico e executor na realização do procedimento, assim como neste estudo, em que apenas 30,9\% assinalaram esta alternativa ${ }^{17}$.

As questões 3 e 11 descrevem a sequência correta da técnica de sondagem vesical de demora, devendo colocar o paciente em decúbito dorsal com as pernas afastadas, realizar lavagem externa da genitália e fixar a sonda na região supra púbica sem tracioná-la ao término. Enquanto as questões 9, 10 e 12 referem-se à alternativa incorreta ou que não faz parte da técnica do cateterismo como colocar campo fenestrado depois da assepsia, conectar a bolsa coletora à sonda de Foley após a retirada do campo fenestrado e calçar luvas de procedimento no final do procedimento. Em 
quase todas essas questões mais da metade dos estudantes demonstraram conhecimento.

Em uma pesquisa, o procedimento de cateterismo urinário padronizado, descrito em algumas instituições no interior do estado de São Paulo, é composto pelo preparo e organização do material, interação enfermeiro/paciente, higienização externa, disposição e abertura do material junto ao paciente, montagem do material utilizado (conexão do cateter vesical junto ao coletor-sistema fechado), antissepsia, cateterização, fixação do cateter e coletor e recomposição da unidade do paciente. A operacionalização das etapas do procedimento foi apresentada de diferentes maneiras, porém a sequência preconizada para a sua realização foi descrita conforme o encontrado nos livros-textos e nas diretrizes que fundamentam a prática de enfermagem sobre $o$ assunto ${ }^{17}$.

A European Association of Urology Nurses (EAUN) descreve em seu documento Evidencebased Guidelines for Best Practice in Urological Health Care Catheterisation que no procedimento de cateterismo em homens é preciso ajudar o paciente a permanecer na posição supina para assegurar que o pênis fique acessível, não expondo o paciente nesta fase e após abrir os materiais, usando técnica asséptica, conectar a bolsa coletora ao cateter para reduzir o risco de infecção cruzada ${ }^{2}$.

Alguns autores apontam que, apesar da ausência de consenso sobre o momento ideal para a conexão da sonda a bolsa coletora, essa prática facilita a execução da técnica, garante o sistema fechado desde a introdução da sonda e impossibilita possível respingo de urina na mucosa ocular. Ainda, destacam que a fixação do cateter pode ser uma medida de prevenção e controle de infecção do trato urinário no cateterismo vesical de longa permanência, sendo a região inguinal ou abdominal inferior de escolha no sexo masculino, para reduzir a curva uretral e eliminar a pressão no ângulo peniano escrotal, o que pode ocasionar fístula, porém essa questão foi apontada como correta nesta pesquisa somente por $25,9 \%$ dos estudantes ${ }^{16}$.

As questões 4 e 8 fazem referência sobre o primeiro passo para evitar uma infecção do trato urinário e a lavagem externa para iniciar o procedimento no sexo masculino. A maioria dos estudantes selecionou a opção correta com a realização da técnica asséptica de inserção do cateter. Contudo, somente $17,3 \%$ apontaram a alternativa com a sequência correta da lavagem externa.

De acordo com o estudo realizado em hospitais de Goiânia com profissionais de enfermagem sobre o conhecimento e adesão ao controle de infecção do cateterismo vesical, observou-se que a higienização das mãos é a mais simples e importante medida na prevenção de infecção, seguida do uso de luvas estéreis e rigorosa realização de técnica asséptica na execução do procedimento. A higiene intima prévia a antissepsia é medida fundamental na prevenção das infecções do trato urinário ${ }^{16}$.

A higiene intima prévia à introdução do cateter urinário é realizada para prevenir a entrada de microrganismos na uretra, pois estes podem aderir-se e criar um anel ao redor do cateter urinário, levando a irritação e fricção do meato. Água morna e sabão devem ser usados, sem causar traumas, de modo que todas as áreas perineais estejam limpas. No final, o sabão deve ser completamente removido e a área deve ser $\operatorname{seca}^{2,17}$.

Nas questões 6 e 7 as alternativas corretas envolvem os cuidados na manutenção do cateter de longa permanência, abordando que não é necessária a rotina de troca da sonda vesical de longa permanência se não houver protocolo específico na instituição e a desconexão do sistema de drenagem da sondagem vesical de demora só deve ser indicada em casos de obstrução e troca deste, respectivamente. Mais da metade dos estudantes acertou a questão 7 , porém poucos colocaram a alternativa correta sobre a rotina de troca de sonda.

Apesar dos autores do questionário aplicado nesta pesquisa colocar como alternativa certa a desconexão do sistema de drenagem da sonda em casos de obstrução, estudos apontam isso como uma prática inadequada. Ao realizar este procedimento, optando-se somente pela troca da bolsa, por não ser uma conduta descrita em protocolo padronizado, impossibilita o seu registro e impede o seu rastreamento, amplia os riscos e não garante a qualidade do atendimento ao paciente ${ }^{18}$. Assim, conforme descrito no Guideline for Prevention of Catheter-Associated Urinary Tract Infections 2009, se houver obstrução e provavelmente o material do cateter esteja contribuindo para esta, deve-se trocar a sonda vesical ${ }^{15}$.

Para evitar a obstrução e manter o fluxo, a bolsa coletora deve ser mantida o abaixo do 
nível da bexiga em todos os momentos, possibilitando a drenagem da urina por gravidade e nunca deve ser repousada no chão ${ }^{11,15}$.

Estudos recomendam a permanência dos cateteres por até $24 \mathrm{~h}$ após a cirurgia para redução das taxas de ITU sintomática. Entretanto, o tempo de permanência do cateter deve ser monitorado de acordo com as condições clínicas do paciente ou sinais que indiquem sua troca (formação de resíduos, vigência de sepsis, febre de origem desconhecida, obstrução da luz do cateter ou tubo coletor, suspeita ou evidências de incrustações no lúmen do cateter, contaminação do cateter por técnica inapropriada na instalação ou no manuseio, desconexão acidental do cateter com o tubo coletor, mau funcionamento, deterioração do cateter, tubo ou saco coletor e piúria visível). Não está pré-estabelecido um período para a troca do cateter vesical e não se recomenda intervalos fixos para a mesma ${ }^{8}$.

A cateterização urinária é um procedimento invasivo e exclusivo do enfermeiro executada no cotidiano de sua prática assistencial. Apesar de ser considerado um procedimento comum, ele está associado a complicações que requerem esforços da enfermagem para seu controle. Dessa forma, torna-se essencial assistência de enfermagem com qualidade e de menor custo, segura e baseada em informações atualizadas e padronizadas $^{8,11}$.

Além disso, é uma das técnicas considerada pelos graduandos de enfermagem como de maior atribuição de estresse. 0 esquecimento da sequência do procedimento de cateterismo vesical e a pequena valorização da mesma resultam em conflito ao profissional recém-formado devido à falta de segurança. $O$ déficit de conhecimento sobre a técnica específica pode culminar em iatrogenias graves e complicações ${ }^{13}$.

Assim, para o processo de ensino e aprendizagem das técnicas, ressalta-se 0 interesse como um facilitador e pelo menos parte desse efeito deve ser atribuída ao conhecimento prévio. A aprendizagem significativa só ocorre quando a informação nova é ligada a conceitos existentes, levando a um processo de conexão que envolve a relação de uma ideia nova com um conceito prévio e, ao mesmo tempo, na modificação de ambos, isto é, dando significado a ambos. Dessa forma, a aprendizagem precisa ser encarada como um processo interno e pessoal, colocando o estudante na construção ativa do conhecimento e que progride no tempo de acordo com os interesses e capacidades individuais ${ }^{19,20}$.

Conclui-se que boa parte dos estudantes do curso de Enfermagem tem conhecimento acerca da técnica do cateterismo vesical de longa permanência, pois na maioria das questões mais da metade do grupo apontou a alternativa correta.

Como limitação do estudo, encontra-se a falta de padronização do procedimento de cateterismo urinário e/ou de garantia de sua aplicação na prática assistencial em instituições de ensino e serviços. Ainda, mesmo com a adaptação realizada pelos pesquisadores, o questionário aplicado pode ter apresentado pontos de abordagem inadequados por não ter sido criado na mesma instituição onde foi aplicado. Apesar das limitações, este estudo apresenta uma visão geral acerca do conhecimento dos estudantes de enfermagem sobre a técnica em cateterismo vesical, auxiliando na adequação e reorganização do ensino das técnicas de enfermagem e fornecendo subsídio para futuras discussões e pesquisas.

\section{CONFLITO DE INTERESSE}

Os autores declaram não haver qualquer potencial conflito de interesse que possa interferir na imparcialidade deste trabalho científico.

\section{REFERÊNCIAS}

1. Cardoso SAC, Maia LFS. Cateterismo vesical de demora na UTI adulto: o papel do enfermeiro na prevenção de infecção do trato urinário. Rev Recien. 2014;4(12):5-14. DOI: http://dx.doi.org/10.24276/rrecien23583088.2014.4.12.5-14

2. European Association of Urology Nurses. Evidence bases guidelines for best practice in urological health care. Catheterisation. Indwelling catheters in adults urethral \& suprapubic. 2012. Acesso em 10 mai 2019. Disponível em: http://www.uroweb.org/guidelines/onlineguidlines

3. Silva JP, Brandão JOC, Medeiros CSQ. Intervenção de Enfermagem na prevenção das infecções do trato urinário relacionado ao cateterismo vesical de demora: uma revisão 
integrativa da literatura. Cad Graduação: Ciên Biol Saúde. 2014;1(3):21-33.

4. Merces $M C$, Carvalho MAM, Araújo PRS, Queiroz AB, Silva BSM, Sousa MNM, Servo MLS. A prática do(a) enfermeiro(a) na inserção do cateter de Folley em pacientes de unidade de terapia intensiva: limites e possibilidades. Rev Epidemiol Control Infect. 2013;3(2):55-61.

5. Conselho Federal de Enfermagem - COFEN. Resolução COFEN no 0450, de 11 de dezembro de 2013. Normatiza o procedimento de sondagem vesical no âmbito do Sistema Cofen / Conselhos Regionais de Enfermagem. Ministério da Saúde. Brasília (DF); 2013. Acesso 30 abr 2019. Disponível em: http:// http://www.cofen.gov.br/resolucao-cofen-no04502013-4 23266.html

6. Santos E, Napoleão AA. Complicações relacionadas ao uso de cateter vesical de demora e o papel da Enfermagem: reflexão. CuidArte Enferm. 2010;4(2):88-91.

7. Igawa $\mathrm{Y}$, Wyndaele JJ, Nishizawa $\mathrm{O}$. Catheterization: possible complications and their prevention and treatment. Int J Urol. 2008;15:481-5.

http://dx.doi.org/10.1111/i.14422042.2008.02075.x

8. Ercole FF, Macieira TGR, Wenceslau LCC, Martins AR, Campos CC, Chianca TCM. Revisão integrativa: evidências na prática do cateterismo urinário intermitente/demora. Rev Latino-Am Enferm. 2013;21(1):459-68. DOI: http://dx.doi.org/10.1590/S010411692013000100023

9. Ribeiro RG, Santos APF, Oliveira FM, Santos K, Godinho EMA. Infecção hospitalar do trato urinário relacionada ao cateterismo vesical de demora. Rev Científica FACS. 2011;13(14):50-7.

10. Jorge BM, Mazzo A, Mendes IAC, Trevizan MA, Martins JCA. Infeção do trato urinário relacionada com o uso do cateter: revisão integrativa. Rev Enferm Ref. 2013;3(11):125-132. DOI: http://dx.doi.org/10.12707/RIII1271

11. Mazzo A, Bardivia CB, Jorge BM, Souza Júnior VD, Fumincelli L, Mendes IAC. Urinary catheterization delay: clinical practice. Enfermería Global. 2015;38:69-77.

12. Balduino LSC. Validação de instrumentos para avaliação da habilidade e do conhecimento acerca da sondagem vesical de demora. [Dissertação]. Universidade Federal do Rio Grande do Norte; Natal, 2013.

13. Evangelista RA, Hortense $P$, Sousa FAEF. Estimação de magnitude do estresse, pelos alunos de graduação, quanto ao cuidado de enfermagem. Rev Latino-Am Enferm. 2004;12(6):913-7. DOI: http://dx.doi.org/10.1590/S0104$\underline{11692004000600010}$

14. Spíndola T, Martins ERC, Francisco MTR. Enfermagem como opção: perfil de graduandos de duas instituições de ensino. Rev Bras Enferm. 2008;61(2):164-9. DOI: http://dx.doi.org/10.1590/S0034$\underline{71672008000200004}$

15. Gould CV, Umscheid CA, Agarwal RK, Kuntz G, Pegues DA. Health Care Infection Control Practices Advisory Committee. Guideline for prevention of cateter associated urinary tract infections 2009. Atlanta, GA: Healthcare Infection Control Practices Advisory Committee; 2009.

16. Souza ACS, Tipple AFV, Barbosa JM, Pereira MS, Barreto RASS. Cateterismo urinário: conhecimento e adesão ao controle de infecção pelos profissionais de enfermagem. Rev Eletr Enferm. 2007;9(3):724-35.

17. Mazzo A, Gaspar AACS, Mendes IAC, Trevizan MA, Godoy S, Martins JCA. Cateter urinário: mitos e rituais presentes no preparo do paciente. Acta Paul Enferm. 2012;25(6): 889-94. DOI: http://dx.doi.org/10.1590/50103$\underline{21002012000600010}$

18. Mazzo A, Godoy S, Alves LM, Mendes IAC, Trevizan MA, Rangel EML. Cateterismo urinário: facilidades e dificuldades relacionadas à sua padronização. Texto Contexto Enferm. 2011;20(2):333-9. DOI: http://dx.doi.org/10.1590/S0104$\underline{07072011000200016}$

19. Tobias S. Interest, prior knowledge, and learning. Rev Educ Res. 1994;14(1):37-64. 
20. Vasconcelos C, Praia JF, Almeida LS. Teorias de aprendizagem e o ensino/aprendizagem das ciências: da instrução à aprendizagem. Psicol Escolar Educ. 2003;7(1):11-9. DOI:

http://dx.doi.org/10.1590/S1413-

85572003000100002 